\title{
BMJ Open Effect of electronic health interventions on metabolic syndrome: a systematic review and meta-analysis
}

\author{
Dandan Chen, ${ }^{1}$ Zhihong Ye (D) , ${ }^{1}$ Jing Shao, ${ }^{2}$ Leiwen Tang, ${ }^{1}$ Hui Zhang, ${ }^{3}$ \\ Xiyi Wang (D) , ${ }^{1}$ Ruolin Qiu, ${ }^{1}$ Qi Zhang ${ }^{1}$
}

To cite: Chen D, Ye Z, Shao J, et al. Effect of electronic health interventions on metabolic syndrome: a systematic review and meta-analysis. BMJ Open 2020;10:e036927. doi:10.1136/ bmjopen-2020-036927

- Prepublication history for this paper is available online To view these files, please visit the journal online (http://dx.doi org/10.1136/bmjopen-2020 036927).

Received 20 January 2020 Revised 09 June 2020 Accepted 22 August 2020
Check for updates

(c) Author(s) (or their employer(s)) 2020. Re-use permitted under CC BY-NC. No commercial re-use. See rights and permissions. Published by BMJ.

${ }^{1}$ Affiliated Sir Run Run Shaw Hospital, Zhejiang University School of Medicine, Hangzhou, China

${ }^{2}$ School of Nursing, Zhejiang University School of Medicine, Hangzhou, Zhejiang, China ${ }^{3}$ Guizhou Provincial People's Hospital, Guiyang, China

Correspondence to Dr Zhihong Ye; yezh@zju.edu.cn

\section{ABSTRACT}

Objective We aimed to examine whether eHealth interventions can effectively improve anthropometric and biochemical indicators of patients with metabolic syndrome (MetS).

Design Systematic review and meta-analysis. Methods PubMed, the Web of Science, Embase, Medline, CINAHL, PsycINFO, the Cochrane Library, the Chinese National Knowledge Infrastructure, the Wanfang and Weipu databases were comprehensively searched for papers that were published from database inception to May 2019. Articles were included if the participants were metabolic syndrome (MetS) patients, the participants received eHealth interventions, the participants in the control group received usual care or were wait listed, the outcomes included anthropometric and biochemical indicators of MetS, and the study was a randomised controlled trial (RCT) or a controlled clinical trial (CCT). The Quality Assessment Tool for Quantitative Studies was used to assess the methodological quality of the included articles. The meta-analysis was conducted using Review Manager V.5.3 software.

Results In our review, seven RCTs and two CCTs comprising 935 MetS participants met the inclusion criteria. The results of the meta-analysis revealed that eHealth interventions resulted in significant improvements in body mass index (standardised mean difference $(\mathrm{SMD})=-0.36,95 \% \mathrm{Cl}(-0.61$ to -0.10$), p<0.01$ ), waist circumference $(S M D=-0.47$, $95 \% \mathrm{Cl}(-0.84$ to -0.09$), \mathrm{p}=0.01)$ and systolic blood pressure(SMD $=-0.35,95 \% \mathrm{Cl}(-0.66$ to -0.04$), \mathrm{p}=0.03)$ compared with the respective outcomes associated with the usual care or wait-listed groups. Based on the included studies, we found significant effects of the eHealth interventions on body weight. However, we did not find significant positive effects of the eHealth interventions on other metabolic parameters.

Conclusions The results indicated that eHealth interventions were beneficial for improving specific anthropometric outcomes, but did not affect biochemical indicators of MetS. Therefore, whether researchers adopt eHealth interventions should be based on the purpose of the study. More rigorous studies are needed to confirm these findings.

\section{INTRODUCTION}

Metabolic syndrome (MetS) represents an important public health problem. MetS has different diagnostic criteria, but it is characterised by at least three of five metabolic

\section{Strengths and limitations of this study}

To the best of the researchers' knowledge, this is the first systematic review and meta-analysis on eHealth interventions for metabolic syndrome patients.

- The strengths of this review included its adherence to the Preferred Reporting Items for Systematic Reviews and Meta-analyses guidelines, the comprehensive literature search and the inclusion of eHealth interventions in a predefined patient sample.

- Another strength of this meta-analysis was that randomised controlled trials (RCTs) and controlled clinical trials (CCTs) were included, which are good standards for evidence-based clinical research.

- The number of RCTs and CCTs and the overall sample size included in the meta-analysis were small.

- We only searched the Chinese and English databases. More high-quality articles should be included.

risk factors: abdominal obesity, elevated triglycerides (TG), reduced high-density lipoprotein cholesterol (HDL-C), hypertension and impaired glucose tolerance. The prevalence of MetS is increasing and is even likely to reach epidemic proportions, which will result in substantial medical costs ${ }^{1}$ and impose a heavy burden on the healthcare system. A previous study indicated that over $20 \%$ of the world's population met the criteria for MetS, and individuals with MetS were three times more likely to develop cardiovascular disease and five times more likely to develop type 2 diabetes mellitus. ${ }^{2}$ Moreover, patients with MetS experienced higher cancer risks ${ }^{3}$ and worse health-related quality of life ${ }^{4}$ than individuals without MetS. In view of the negative outcomes of MetS, it is necessary to identify and control risk factors for MetS. Factors such as older age, female sex,${ }^{5}$ stress, ${ }^{6}$ low physical activity, ${ }^{7}$ overweight or obesity, ${ }^{8}$ high waist circumference (WC), elevated TG, elevated fasting blood glucose (FBG) and high average diastolic blood pressure $(\mathrm{DBP})^{9}$ exhibited a close relationship with the progression of 


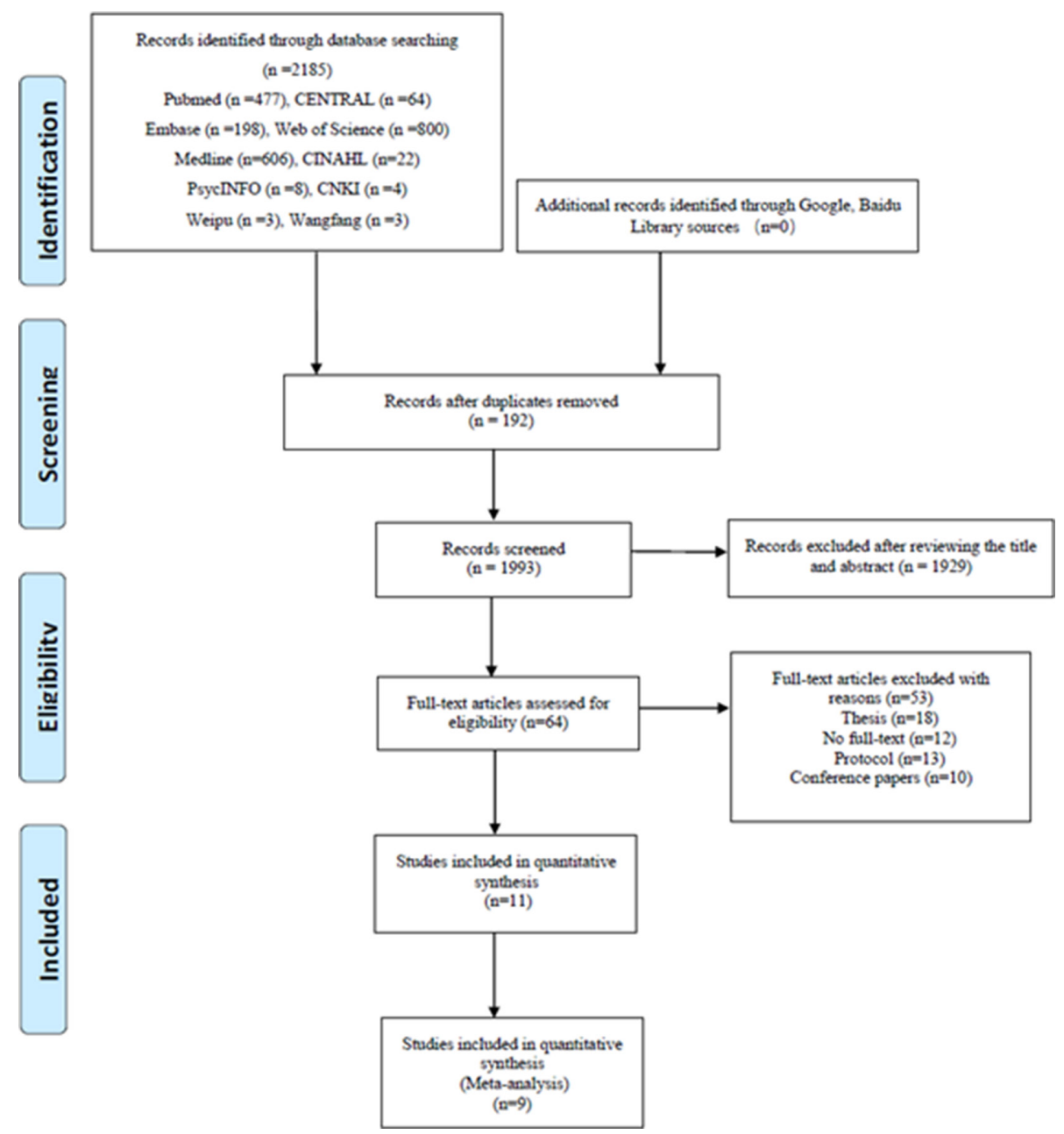

Figure 1 Flow chart of article selection process. CNKI, Chinese National Knowledge Infrastructure.

MetS. Therefore, healthcare professionals should take measures to effectively manage and treat MetS.

Pharmacological therapy and lifestyle interventions are commonly employed to prevent and treat MetS. ${ }^{10}$ However, drugs sometimes have adverse effects and are accompanied by limited efficacy. ${ }^{11}$ Therefore, researchers pay more attention to lifestyle interventions, which focus on increasing physical activity and improving the diet, and these interventions could reduce MetS risks. ${ }^{12}{ }^{13}$ In the healthcare system, interest in the application of eHealth devices to conduct lifestyle interventions for patients is growing. eHealth refers to 'health services and information delivered or enhanced through the internet and related technologies', ${ }^{14}$ which includes internet and computer, mobile phone (the use of text messaging and applications on mobile phones), telehealth, electronic monitors and wireless and Bluetooth enabled devices. ${ }^{15}$ eHealth interventions have become increasingly popular due to making treatments more accessible and affordable. ${ }^{16}{ }^{17}$ They provide benefits for patients with an inconvenient location or commute and unavailable or inflexible times, and patients may receive the required information in a cost-effective way.

The increased use of eHealth devices may create new opportunities to manage MetS in the coming years. A study by Jahangiry et $a l^{18}$ found that eHealth interventions, such as web-based interventions, could significantly improve physical activity, dietary intake and several dimensions of quality of life among MetS patients. Furthermore, eHealth interventions were promising approaches to reduce health-related stress in MetS patients ${ }^{19}$ and could also provide patients with real-time feedback and with tailored interventions according to their needs. ${ }^{20}$ Therefore, eHealth interventions may be more convenient, more flexibly fitted to patients' needs and promote greater treatment adherence.

While the growing benefits of eHealth are evident, researchers have found that the effects of eHealth interventions on anthropometric and biochemical indicators of MetS were not consistent. Although two systematic reviews have reported the positive effects of eHealth interventions on blood pressure ${ }^{21}$ and blood glucose,${ }^{22}$ the two studies did not target patients with MetS. Given that a single study cannot disprove the effects of eHealth interventions among MetS patients and none of the systematic reviews based on randomised controlled trials (RCTs) and controlled clinical trials (CCTs), which are associated with rigorous study design, have been conducted to explore the efficacy of eHealth interventions on anthropometric and biochemical indicators of MetS patients, the primary objective of this review is to determine whether eHealth 


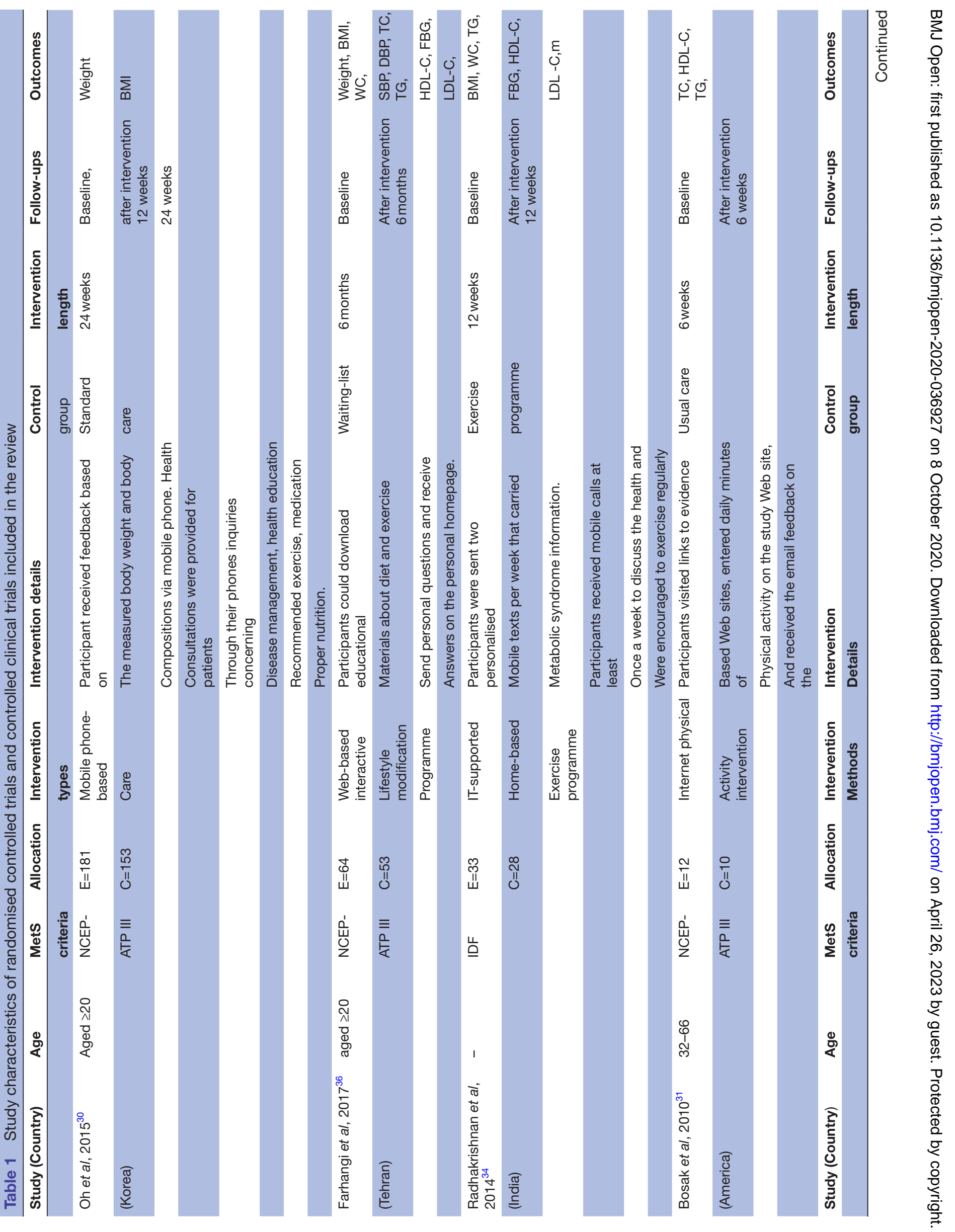




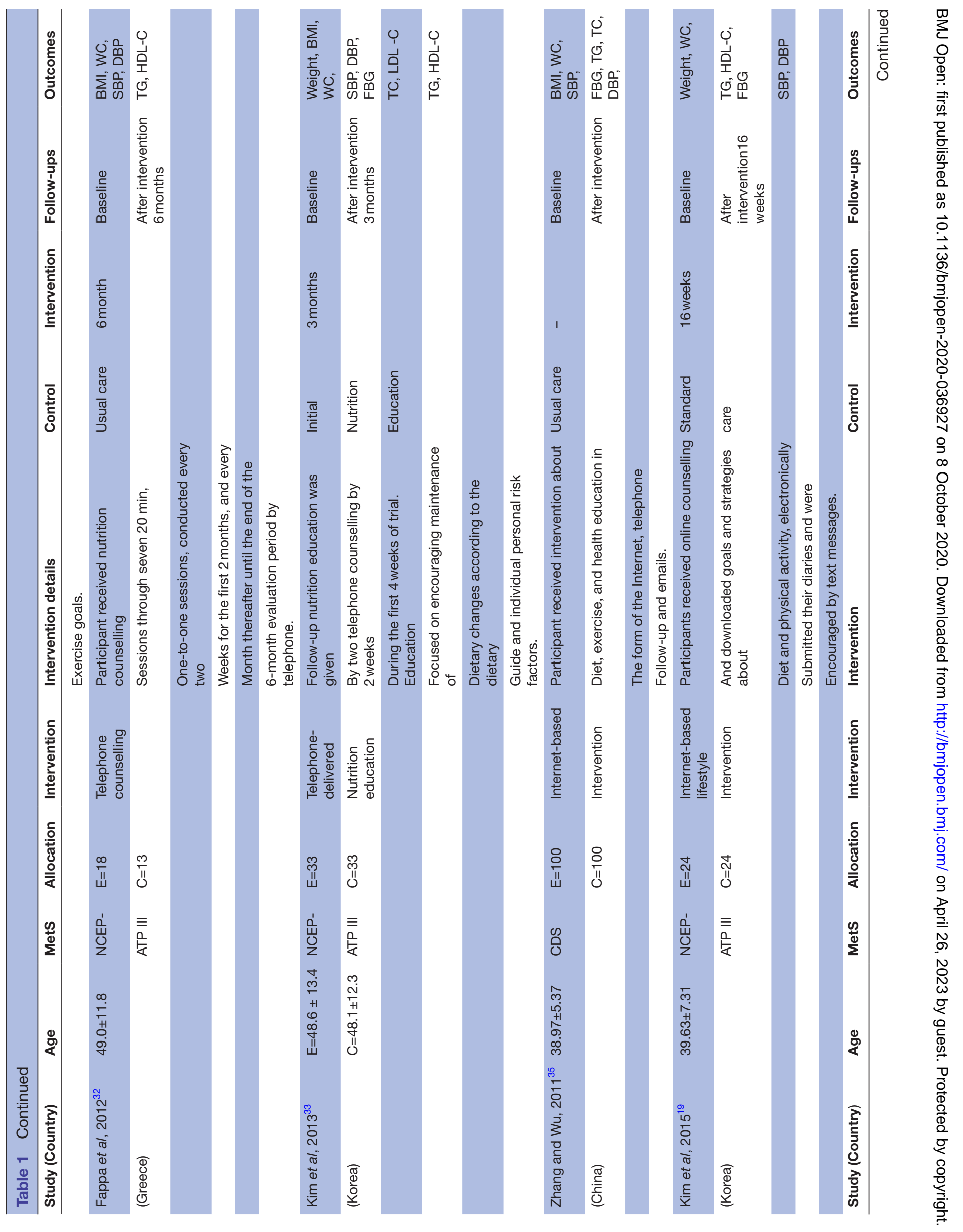


interventions are effective at improving anthropometric and biochemical indicators of MetS among patients with MetS. This finding would not only answer whether eHealth interventions are effective for MetS patients, but also provide a reference point in healthcare communication and promotion using new information technology.

\section{METHODS}

The review was performed according to the Preferred Reporting Items for Systematic Reviews and Meta-analyses guidelines. ${ }^{23}$

\section{Literature search}

PubMed, the Web of Science, Embase, the Cochrane Library, Medline, CINAHL, PsycINFO, the Chinese National Knowledge Infrastructure, the Wanfang and Weipu databases were systematically searched for papers that were published through May 2019. The search terms were conjunctions of the following terms: "mobile applications" OR "mobile application" OR "mobile apps" OR "mobile app" OR "cell phones" OR "cell phone" OR "smartphone" OR "text messaging" OR "text message" OR "mobile phones" OR "mHealth" OR "mobile health" OR "internet" OR "web" OR "eHealth" OR "online interventions" OR "telehealth" OR "telephone" OR "SMS" OR "short message" OR "mobile technology" AND "metabolic syndrome" OR "Metabolic Syndromes" OR "syndrome, metabolic" OR "syndromes, metabolic" OR "MetS". In addition, manual searches of cited references in relevant papers were conducted if appropriate. Missing relevant articles were obtained by contacting authors. An example of the PubMed search terms can be found in online supplemental file 1 .

\section{Study selection}

The inclusion criteria of this review were as follows: (1) participants: patients with a clinical diagnosis of MetS. The diagnosis of MetS was performed using the International Diabetes Federation (IDF) and National Cholesterol Education Programme Adult Treatment Panel III (NCEP ATP III) or criteria closely aligned to these definitions prior to publication of these definitions in 2001; (2) interventions: patients with MetS received eHealth interventions; (3) comparisons: the participants in the control group received usual care or were wait listed; (4) outcomes: anthropometric and biochemical indicators, including body weight, body mass index (BMI), WC, systolic blood pressure (SBP), DBP, FBG, total cholesterol (TC), HDL-C, low-density lipoprotein cholesterol (LDLC), TG or fasting insulin and (5) study designs: RCTs or CCTs. The exclusion criteria were as follows: (1) studies that were literature reviews, qualitative studies or protocols; (2) studies in which participants were not patients with MetS; (3) studies in which the intervention methods were not eHealth interventions; (4) studies that did not report anthropometric or biochemical indicators and (5) publications that were not in English or Chinese. 
Table 2 Assessment for the methodological quality of the included studies

\begin{tabular}{|c|c|c|c|c|c|c|c|}
\hline Author, Year & $\begin{array}{l}\text { Selection } \\
\text { bias }\end{array}$ & $\begin{array}{l}\text { Study } \\
\text { design }\end{array}$ & Confounders & Blinding & $\begin{array}{l}\text { Data } \\
\text { collection }\end{array}$ & Withdrawals & $\begin{array}{l}\text { Global } \\
\text { rating }\end{array}$ \\
\hline & & & & & Methods & And drop-out & \\
\hline Farhangi et al, $2017^{36}$ & Moderate & Strong & Weak & Moderate & Strong & Moderate & Moderate \\
\hline $\begin{array}{l}\text { Radhakrishnan et al, } \\
2014^{34}\end{array}$ & Moderate & Strong & Weak & Moderate & Strong & Strong & Moderate \\
\hline Fappa et al, $2012^{32}$ & Strong & Strong & Strong & Moderate & Strong & Weak & Moderate \\
\hline Kim et al, $2013^{33}$ & Strong & Strong & Strong & Moderate & Strong & Strong & Strong \\
\hline Zhang and Wu $2011^{35}$ & Moderate & Strong & Strong & Moderate & Strong & Moderate & Strong \\
\hline Kim et al, $2015^{19}$ & Strong & Strong & Strong & Moderate & Strong & Strong & Strong \\
\hline
\end{tabular}

Two authors (DC and JS) independently screened the titles and abstracts of all potentially relevant studies. We ultimately identified the papers that met the above described eligibility criteria and obtained the full text of these articles for this systematic review and meta-analysis. Discussion was used to resolve differences.

\section{Data extraction}

The data were extracted from the included articles using standardised extraction forms. Data included age, country, diagnostic criteria, the number of participants in the experimental and control groups, intervention methods and details, control details, duration of interventions, follow-ups and outcomes. Two authors (DC and JS) independently extracted data from each study, and inconsistencies were resolved through discussion with a third author (ZY). Authors of these studies were contacted if more data were needed. Data extraction form could be found in online supplemental file 2 .

\section{Quality assessment}

The methodological quality of all studies was measured using the Quality Assessment Tool for Quantitative Studies, developed by the Effective Public Health Practice Project, Canada. This tool could be used for RCTs, quasi-experimental studies and uncontrolled studies. The content and construct validity have been established. ${ }^{24}$ Two authors (DC and HZ) independently assessed the quality of the included studies. Studies were assessed based on six criteria: selection bias, study design, confounders, blinding, data collection methods and withdrawals and drop-out. The quality rating for the included studies was 'strong', 'moderate' or 'weak'. If the two reviewers disagreed, a third author was available for discussion.

\section{Statistical analysis}

Rev Man V.5.3 software (The Nordic Cochrane Center, The Cochrane Collaboration) was used to quantify the outcomes of the eHealth interventions. Mean net change was used to generate results for this meta-analysis for continuous variables. Mean net changes were calculated as the differences in the changes (mean value postintervention minus mean value at baseline) for both the experimental group and the control group. Intervention effects were measured by the standardised mean difference (SMD) or weighted mean difference with 95\% CIs of standardised mean net changes between the intervention and control groups. ${ }^{25} \mathrm{SMD}$ was interpreted based on Cohen's definitions: $0.2-0.5$ is defined as a small effect, $0.5-0.8$ is a moderate effect and $>0.8$ is a large effect. ${ }^{26}$ The $\mathrm{I}^{2}$ statistic was performed to analyse heterogeneity. An $\mathrm{I}^{2}$ of $25 \%-50 \%$ indicated moderate heterogeneity, and $>50 \%$ indicated high heterogeneity. ${ }^{27}$ Tests of heterogeneity were used to decide which method would be used to obtain the pooled results. When $\mathrm{I}^{2}$ was $>50 \%$, a

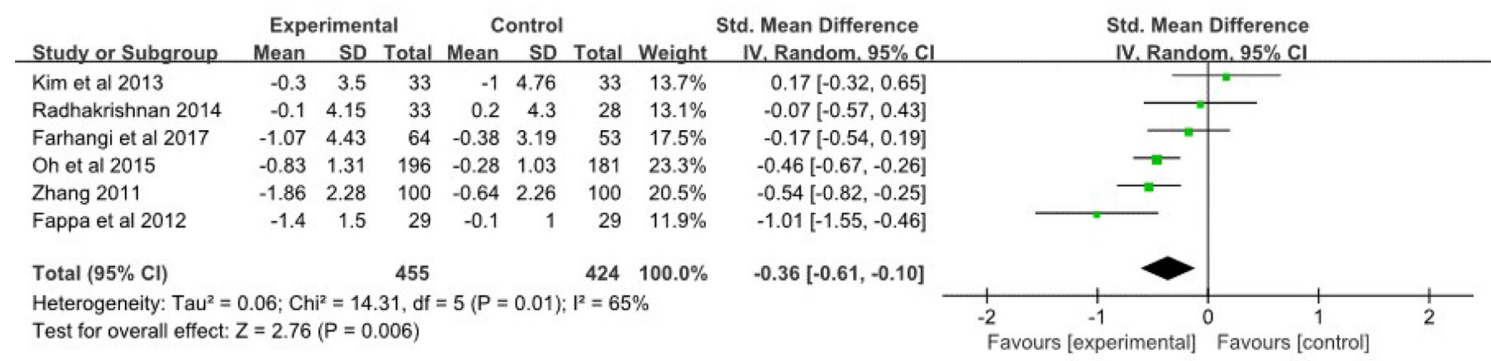

Figure 2 Forest plot for effect of eHealth interventions on standardised mean net changes of BMI. BMI, body mass index; IV, inverse variance. 


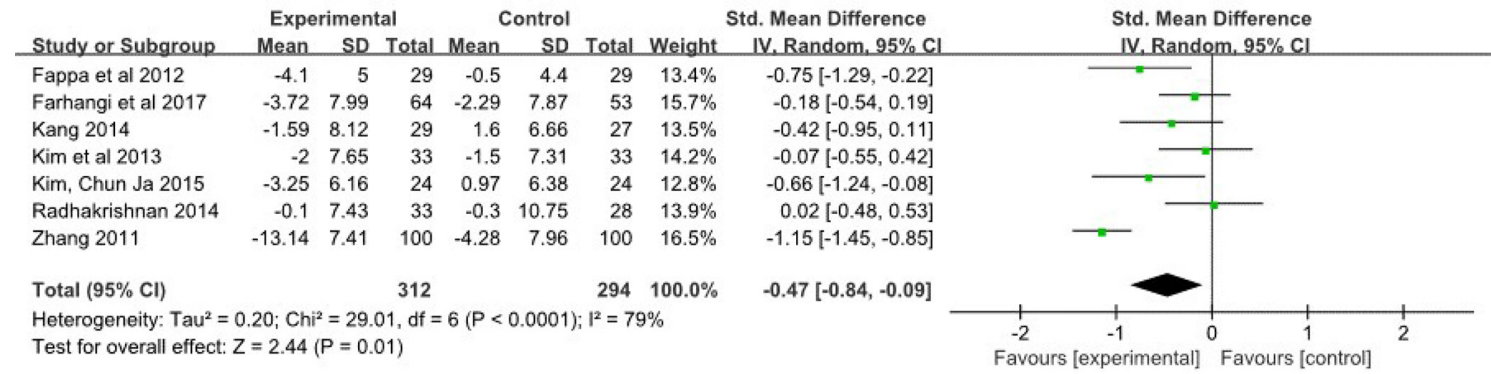

Figure 3 Forest plot for effect of eHealth interventions on standardised mean net changes of WC. WC, waist circumference.

random-effect model was used; otherwise, a fixed-effect model was employed. Significance was defined as $\mathrm{p}<0.05$. Where statistical heterogeneity was detected, possible contributing factors were investigated in sensitivity analyses.

\section{Patient and public involvement}

Patients and the public were not involved in this review.

\section{RESULTS}

Figure 1 illustrates the selection process. The authors retrieved 2185 articles from the databases at the beginning of the study. A total of 1993 records were screened for inclusion after removing 192 repeated documents. Eleven studies matched the above eligibility criteria for the systematic review. For two trials, ${ }^{28} 29$ the original data for meta-analysis could not be obtained. Therefore, seven RCTs $^{30-36}$ and two CCTs ${ }^{19}{ }^{37}$ fulfilled all inclusion criteria for the meta-analysis.

\section{Study characteristics}

Table 1 summarises the information about the characteristics of the included studies. A total of 935 patients with MetS were included in this study. Nine articles were published from 2010 to 2017. Sample sizes ranged from 22 to 200. Most of the studies were performed in developing countries, such as Korea, Tehran, India and China, and two studies were conducted in developed countries, such as the USA and Greece. Moreover, only Oh $e t a l^{30}$ reported more than one follow-up time point. The anthropometric and biochemical indicators were measured using a measuring tape, bioelectrical impedance analysis device, mercury sphygmomanometer and automatic blood analysers. Most studies used the diagnostic criteria of NCEP ATP III, ${ }^{19}{ }^{30-33}{ }^{36}$ Radhakrishnan et $a l,{ }^{34}$ Zhang and $\mathrm{Wu},{ }^{35}$ and Kang et $a l^{37}$ based on the criteria of the IDF, Chinese Diabetes Society and American Heart Association/National Heart, Lung and Blood Institute/International Atherosclerosis Society/International Association for the Study of Obesity/World Heart Federation.

\section{Characteristics of eHealth interventions}

The intervention durations ranged from 6 weeks to 6 months. The types of eHealth interventions were mobile phone-based care interventions $(\mathrm{n}=2),{ }^{3034}$ web-based interactive lifestyle modification programmes $(n=5)^{19} 31$ 35-37 and telephone-delivered interventions $(n=2){ }^{32} 33$ In terms of mobile phone-based care interventions, participants in the intervention groups could inquire about health information and immediately receive feedback that provided MetS information via mobile phone. ${ }^{30}{ }^{34} \mathrm{In}$ addition, five studies ${ }^{19}{ }^{31}$ 35-37 conducted web-based interventions, and the intervention groups could download educational materials about diet and exercise, send personal questions and receive answers on their personal internet homepage. Moreover, Kim et al, ${ }^{33}$ and Fappa et $a l,^{32}$ tested the feasibility of telephone counselling. Participants received telephone counselling sessions and were encouraged to maintain dietary changes according to the dietary guide and individual personal risk factors.

\section{Study quality}

Quality assessments are shown in table 2. In terms of study design and data collection methods, the included studies had high methodologic quality. However, blinding of the participants and assessors who delivered the treatment interventions was not feasible because they could easily identify the treatment. In particular, three studies did not report whether there were differences between the

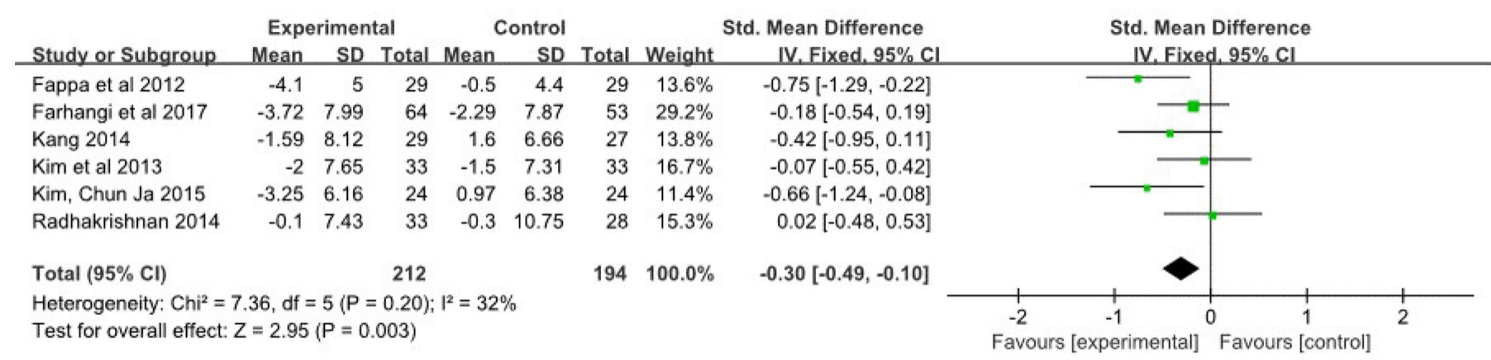

Figure 4 Forest plot for effect of eHealth interventions except from Zhang and Wu study on WC. WC, waist circumference. 


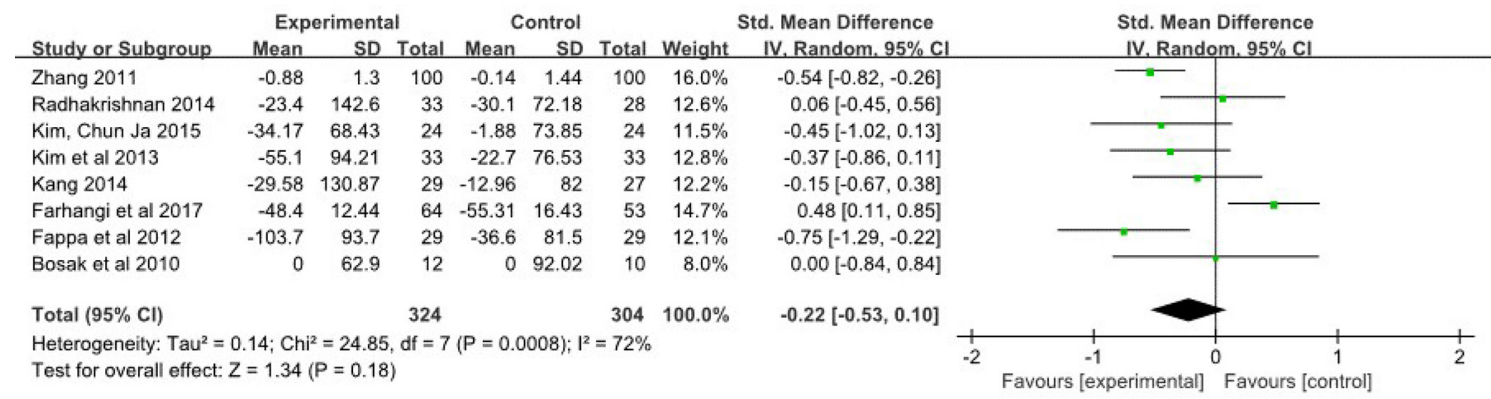

Figure 5 Forest plot for effect of eHealth interventions on standardised mean net changes of TG. TG, triglycerides.

groups at baseline. ${ }^{30} 3436$ These factors influenced the quality of the included studies. Overall, over half of the studies were of high methodological quality.

\section{Effectiveness of eHealth Interventions among patients with MetS}

Body weight

In our study, three included studies chose body weight as an outcome. ${ }^{19} 3033$ Due to the limited number of studies, we chose to describe the results for body weight and did not perform a quantitative summary. Kim et $a l^{19}$ found that there were significant group by time interactions in regard to body weight $(\mathrm{p}=0.022)$. In a study by $\mathrm{Oh}$ et $a l^{30}$ participants in the intervention group showed significant improvements in body weight compared with the body weight in the control group $(\mathrm{p}<0.001)$. Similarly, Kim $e t$ $a \hat{l}^{33}$ reported that at the end of the trial, the intervention group showed a significantly greater reduction in weight than the other group $(\mathrm{p}<0.05)$. Therefore, eHealth interventions may be effective in improving body weight in patients with MetS.

\section{Body mass index}

A meta-analysis of six studies with 879 participants found a significant effect on BMI in the experimental group versus the control group $(\mathrm{SMD}=-0.36,95 \%$ CI $(-0.61$ to $-0.10), \mathrm{p}<0.01)$, with a small effect size pooled across studies. There was substantial evidence of high heterogeneity ( $\mathrm{p}=0.01, \mathrm{I}^{2}=65 \%$ ) (figure 2 ).

\section{Waist circumference}

WC was mentioned as an outcome measurement in seven studies comprising 606 participants. Significant improvements were observed in the experimental groups in comparison with the control groups $(\mathrm{SMD}=-0.47,95 \% \mathrm{CI}(-0.84$ to -0.09$), \mathrm{p}=0.01)$, with a small effect size pooled across studies. There was substantial evidence of high heterogeneity $(p<0.0001$, $\mathrm{I}^{2}=79 \%$ ) (figure 3 ). Zhang and Wu's ${ }^{35}$ study showed the highest effect sizes for WC in the included studies. To explore the source of this considerable heterogeneity, we excluded the Zhang and $\mathrm{Wu}^{35}$ study and found that the heterogeneity decreased $\left(\mathrm{I}^{2}=32 \%\right)$, which indicated that the study contributed to the considerable heterogeneity (figure 4).

\section{Triglycerides}

The impact of eHealth interventions on TG among patients with MetS has been explored in eight studies. As shown in figure 5 , compared with the control groups, the participants who received mHealth and eHealth interventions did not experience significant changes in TG $(\mathrm{SMD}=-0.22,95 \% \mathrm{CI}(-0.53$ to 0.10$), \mathrm{p}=0.18)$. There was substantial evidence of high heterogeneity $(\mathrm{p}=0.0008$, $\mathrm{I}^{2}=72 \%$ ) (figure 5).

\section{Total cholesterol}

In the five reviewed studies, the results suggested that there were no significant differences between the intervention groups and the control groups in regard to TC $(\mathrm{SMD}=0.15,95 \% \mathrm{CI} \quad(-0.20$ to 0.50$), \mathrm{p}=0.39)$. There was evidence of high heterogeneity $\left(\mathrm{p}=0.02, \mathrm{I}^{2}=66 \%\right)$ (figure 6).

\section{High-density lipoprotein cholesterol}

In our review, seven studies found that eHealth interventions did not cause significant effects on HDL-C compared with the effects in the control groups $(\mathrm{SMD}=-0.17,95 \%$ CI ( -0.36 to 0.02$), p=0.09)$. There was no evidence of high heterogeneity $\left(\mathrm{p}=0.68, \mathrm{I}^{2}=0 \%\right)$ (figure 7 ).

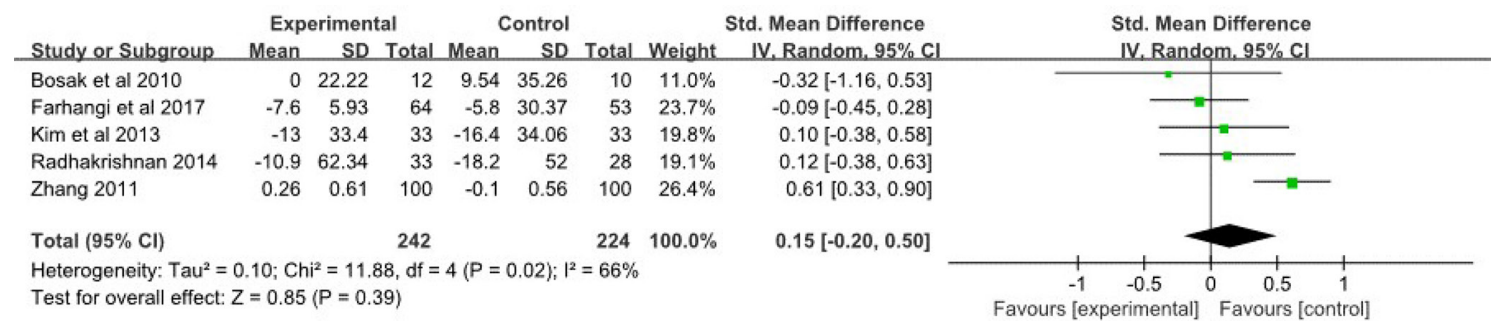

Figure 6 Forest plot for effect of eHealth interventions on standardised mean net changes of TC. TC, total cholesterol. 


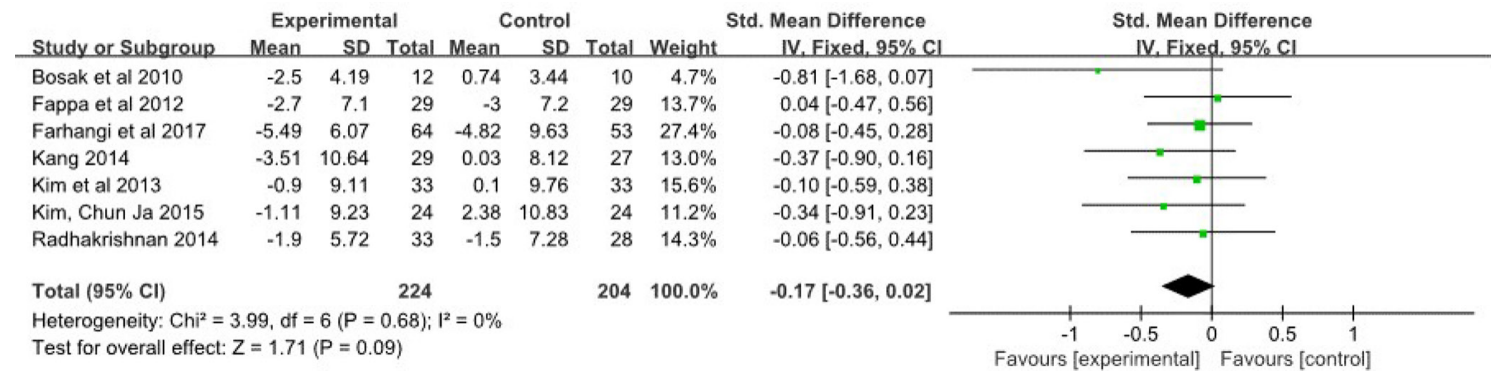

Figure 7 Forest plot for effect of eHealth interventions on standardised mean net changes of HDL-C. HDL-C, high-density lipoprotein cholesterol.

\section{Low-density lipoprotein cholesterol}

In our study, four reviewed studies reported the LDL-C outcome. ${ }^{31333436}$ Farhangi $e t a l^{36}$ found that there were no significant differences in LDL-C between the intervention and control groups. Bosak $e t a l^{31}$ reported no significant effect of an internet intervention on LDL-C compared with the effect observed in the control group. In the study by Kim et $a l,{ }^{33}$ the authors did not find statistically significant effects of the intervention on LDL-C. Moreover, Radhakrishnan et $a l,{ }^{34}$ did not find a significantly positive effect of the intervention on LDL-C compared with of the effect observed in the usual care group.

\section{Systolic blood pressure}

In terms of SBP, six studies found that eHealth interventions had significant effects on SBP (SMD $=-0.35,95 \%$ CI ( -0.66 to -0.04$), \mathrm{p}=0.03)$, with a small effect size pooled across studies. High heterogeneity was detected in the analysis $\left(\mathrm{p}=0.01, \mathrm{I}^{2}=66 \%\right)$ (figure 8$)$.

\section{Diastolic blood pressure}

DBP was only evaluated in six studies. Compared with the control groups, improvements in DBP were not observed in the experimental groups ( $\mathrm{SMD}=-0.35,95 \%$ CI $(-0.82$ to 0.13$), \mathrm{p}=0.15)$. High heterogeneity was found in the meta-analysis of DBP $\left(\mathrm{p}<0.001, \mathrm{I}^{2}=86 \%\right)$ (figure 9). After excluding Zhang and $\mathrm{Wu}^{35}$ study, the heterogeneity decreased $\left(\mathrm{I}^{2}=71 \%\right)$, which indicated that the study contributed to the high heterogeneity (figure 10).

\section{Fasting blood glucose}

As shown in figure 11, in the seven reviewed studies, there were no significant differences in FBG in the control groups and the intervention groups
$(\mathrm{SMD}=-0.27,95 \% \mathrm{CI}(-0.72$ to 0.19$), \mathrm{p}=0.25)$. There was no evidence of high heterogeneity $\left(\mathrm{p}<0.001, \mathrm{I}^{2}=86 \%\right)$ (figure 11). After excluding the Zhang and $\mathrm{Wu}^{35}$ study, the heterogeneity decreased $\left(\mathrm{I}^{2}=0 \%\right)$, which indicated that the study was the origin of the high heterogeneity (figure 12).

\section{Fasting insulin}

In the two reviewed studies, Farhangi et $a l^{36}$ found a significant positive effect of the intervention on fasting insulin compared with the effect observed in the usual care group. However, in the study of Kim et $a l,{ }^{33}$ the results showed no significant differences in fasting insulin between the control groups and the intervention groups.

\section{Summary of results}

As shown in table 3, this systematic review and metaanalysis demonstrated that eHealth interventions resulted in significant improvements in BMI, WC and SBP. However, we did not find significantly positive effects of the eHealth interventions on TG, TC, HDL-C, DBP or FBG compared with the effects observed in the usual care groups or wait listed groups. Moreover, due to the limited number of studies, we could not quantify body weight, LDL-C or fasting insulin. Through the descriptions of the included studies, we found significant effects of the eHealth interventions on body weight. The effects of the eHealth interventions on fasting insulin were mixed, and the effects on LDL-C were negative in the experimental groups compared with the effects in the control groups.

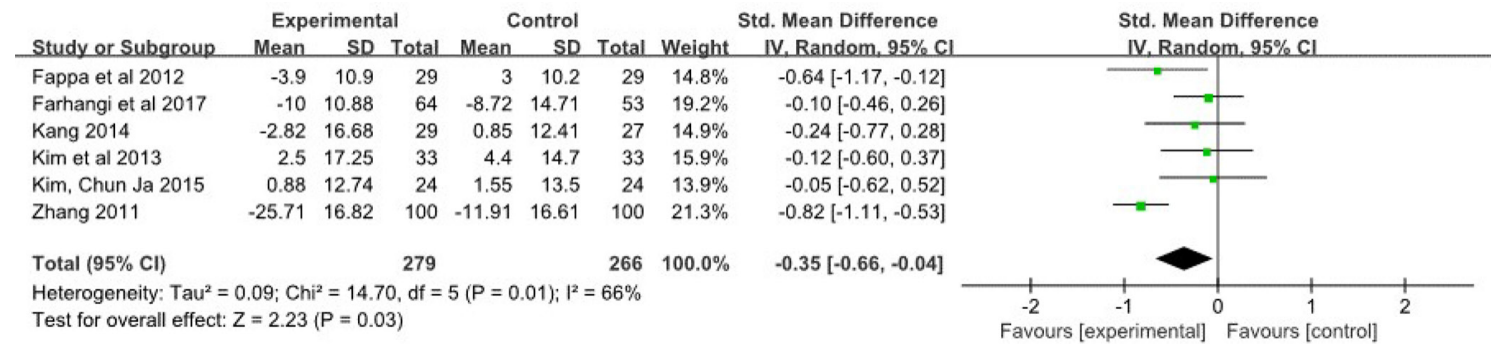

Figure 8 Forest plot for effect of eHealth interventions on standardised mean net changes of SBP. SBP, systolic blood pressure. 


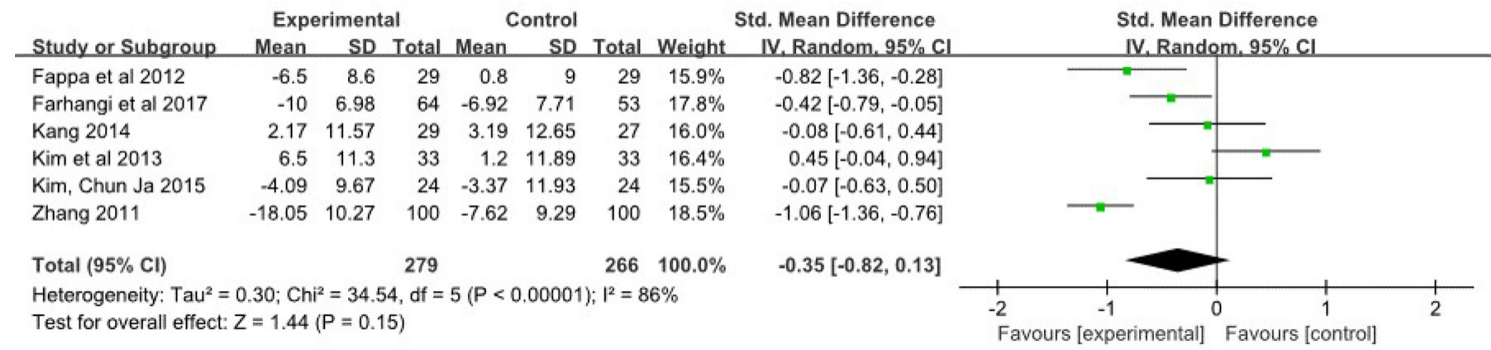

Figure 9 Forest plot for effect of eHealth interventions on standardised mean net changes of DBP. DBP, diastolic blood pressure.

\section{DISCUSSION}

This review is the first to describe and evaluate nine RCTs and CCTs that used eHealth interventions to improve metabolic risk factors among patients with MetS. The current study showed that eHealth interventions resulted in significant improvements in body weight, BMI, WC and SBP compared with the effects of usual care but did not affect TG, TC, HDL-C, LDL-C, FBG or fasting insulin levels. Our results indicated that eHealth interventions were effective as interventions in improving specific anthropometric parameters among individuals with MetS.

Our results showed significant reductions in body weight, $\mathrm{BMI}$ and $\mathrm{WC}$, which was in line with the suggested significant benefits of eHealth interventions in regard to body weight, BMI and WC in the two studies. ${ }^{38}{ }^{39}$ Weight loss is the cornerstone of MetS management. ${ }^{40}$ Weight loss has beneficial impacts on MetS. ${ }^{41}$ The magnitude of weight loss was associated with dose-effect improvements in high blood pressure, hyperglycaemia and hyperlipidaemia. ${ }^{42}$ In contrast, obesity is a risk factor for MetS. ${ }^{30}$ The positive effect on weight loss and BMI might be due not only to the intervention contents focusing on healthy diet and regular physical activity in the included studies, which were the most effective methods for managing MetS, ${ }^{43}$ but also to the fact that the exchange of diet and exercise information through eHealth devices, such as the Internet, was found to be more effective in weight loss and maintenance than traditional methods of self-management. ${ }^{44}$ Kim $e t a \hat{l}^{33}$ found that counselling by telephone could be effective in providing advice and education for MetS patients who need continuous improvement in health behaviours. Moreover, for healthcare professionals, maintaining frequent contact with participants was critical for participant engagement in interventions and to ensure that participants received an adequate intervention. ${ }^{31}$ Additionally, the greatest adherence to lifestyle goals was observed in the eHealth intervention group, which could explain the weight reduction result. ${ }^{32}$ For the WC outcome, a $1 \mathrm{~cm}$ increase in WC increases the risk of cardiovascular events by $2 \%{ }^{45}$ Therefore, WC control is vital for MetS patients. The significant reduction in WC may be attributed to the usefulness of continuous counselling through eHealth devices in the treatment of MetS. ${ }^{33}$ The eHealth devices provided opportunities for patients and medical personnel to communicate, which helped MetS patients have access to health information and improve compliance with interventions. Therefore, a positive effect was observed for WC.

Moreover, in this study, the greater pooled improvements reached significance for SBP. Our result was consistent with those of the studies by Zha ${ }^{46}$ Haas $e t a t^{77}$ and Nolan $e t a l,{ }^{48}$ which illustrated that eHealth interventions effectively improved the level of SBP. This effect might be because eHealth interventions could be more flexibly fitted to MetS patients' lives and promote greater adherence to the lifestyle programme. ${ }^{32}$ In addition, the advantages of eHealth interventions, such as widespread appeal, accessibility, ability to reach large and geographically diverse populations and great compliance at a low cost, ${ }^{49}{ }^{50}$ contributed to the beneficial change.

However, it is also important to note that the eHealth interventions do little to nothing to significantly improve TG, TC, DBP, FBG, HDL-C, LDL-C and fasting insulin. The results from this systematic review were similar to those reported in previous systematic reviews. ${ }^{51}{ }^{52}$ The reason behind this phenomenon may be the differences in study designs, characteristics of study populations, different technologies used in eHealth interventions and

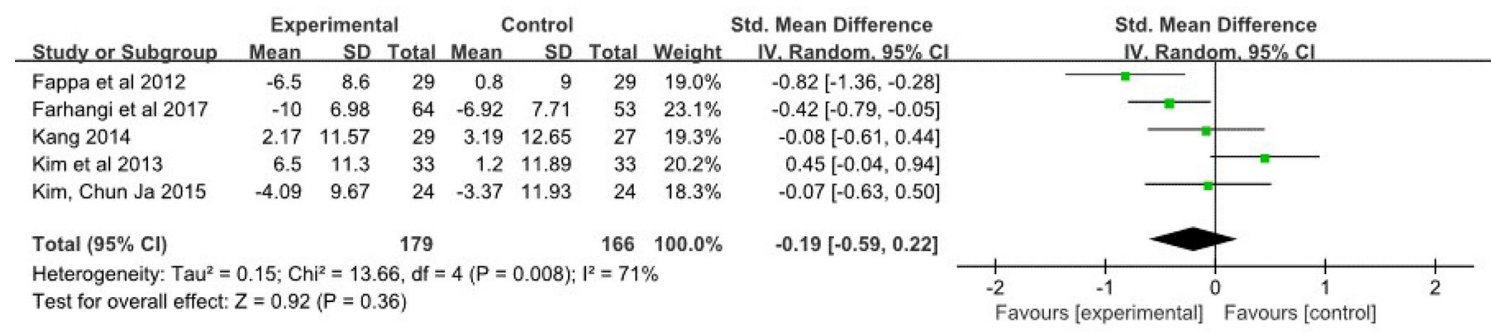

Figure 10 Forest plot for effect of eHealth interventions except from Zhang and Wu study on DBP. DBP, diastolic blood pressure. 


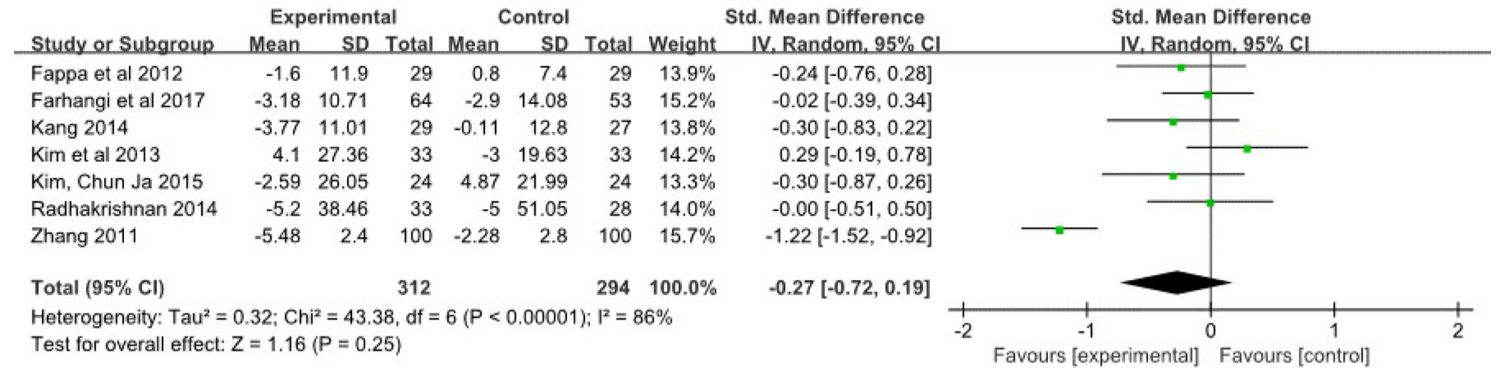

Figure 11 Forest plot for effect of eHealth interventions on standardised mean net changes of FBG. FBG, fasting blood glucose.

duration of the interventions. For instance, the duration of the intervention (6weeks or 8 weeks) in some studies might not be enough to improve many metabolic parameters. ${ }^{37}$ In addition, MetS is characterised by the presence of at least three of five indices. However, these indicators are not homogeneous in patients, as any three of these five indicators are acceptable for the diagnostic criteria of MetS. Therefore, when participants met the inclusion criteria, their normal and abnormal data could be included in the analysis. As a result, the intervention effects may be affected. Additionally, a limited number of studies could explain the nonsignificant changes. We included a total of seven RCTs and two CCTs. More studies should be included to further verify the results.

\section{Limitations}

Several limitations should be acknowledged. First, the number of RCTs and CCTs and the overall sample size included in the meta-analysis were small. Therefore, the findings of our review should be interpreted with caution. Second, because of the limited number of papers, we could not reliably assess publication bias of the included studies and have not explored which eHealth type is more effective. Third, we only searched Chinese and English databases. More high-quality articles should be included. Another limitation of the review was that the results of the meta-analysis had high heterogeneity. Possible sources of heterogeneity included differences in diagnostic criteria for MetS and eHealth types. Finally, most studies were conducted in developing countries, and the majority were performed in Asia. Only two studies were conducted in developed countries. In the future, we should include more target populations from different cultural contexts to increase the representativeness and stability of the results.

Implications for Practice and Future Research eHealth is developing as technology advances and has the potential to provide health communication and promotion. Our study adds value to the current literature as the first systematic review and meta-analysis to provide eHealth interventions for MetS patients. The results indicated that eHealth interventions could be used to improve specific anthropometric and biochemical outcomes among MetS patients, such as body weight, BMI, WC and SBP. However, eHealth interventions could not reduce overall health risks. Due to the limited number of studies, more studies are needed to confirm these results. The eHealth interventions employed a broad range of technologies; however, user satisfaction and adherence to long-term interventions ( $>6$ months) are still unclear. Future studies should focus on user satisfaction with eHealth devices and compliance with long-term interventions, which are important factors affecting the effectiveness of interventions. Additionally, MetS is composed of different components and diverse combination types. However, precise interventions for patients with different metabolic components have not been developed. Therefore, in the future, the optimisation of existing interventions is needed to achieve precise treatment for patients with MetS. This optimisation could be beneficial to healthcare providers trying to recommend an intervention therapy for patients with different characteristics. Moreover, the results of this current study were derived from a small number of RCTs and CCTs, and we believe that more regions and larger sample studies are needed

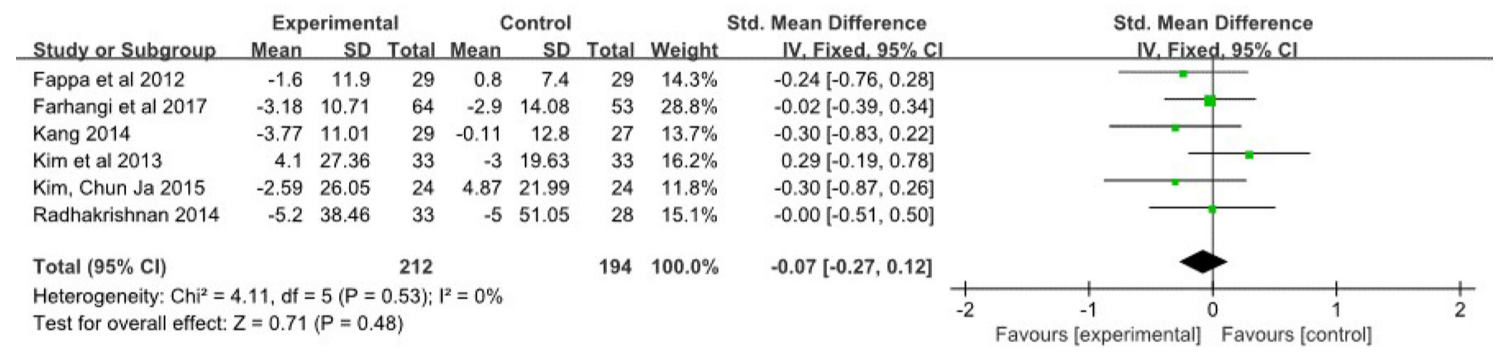

Figure 12 Forest plot for effect of eHealth interventions except from Zhang and Wu study on FBG. FBG, fasting blood glucose. 
Table 3 Estimations of the SMD or MD of related indictors with $95 \% \mathrm{Cl}$ between the intervention and control groups

\begin{tabular}{|c|c|c|c|c|c|}
\hline Variables & $\begin{array}{l}\text { No of } \\
\text { included }\end{array}$ & $\begin{array}{l}\text { SMD (random effect) or } \\
\text { MD (fixed effect) }\end{array}$ & $95 \% \mathrm{Cl}$ & $I^{2}(\%)$ & $\begin{array}{l}P \\
\text { value }\end{array}$ \\
\hline Body mass index & Six & -0.36 & -0.61 to -0.10 & 65 & $0.006^{*}$ \\
\hline Triglycerides & Eight & -0.22 & -0.53 to 0.10 & 72 & 0.18 \\
\hline Total cholesterol & Five & 0.15 & -0.25 to 0.50 & 66 & 0.39 \\
\hline Diastolic blood pressure & Six & -0.35 & -0.82 to 0.13 & 86 & 0.15 \\
\hline Fasting blood glucose & Seven & -0.27 & -0.72 to 0.19 & 86 & 0.25 \\
\hline
\end{tabular}

${ }^{*} \mathrm{P}<0.05$.

$\mathrm{MD}$, mean difference; SMD, standardised mean difference.

before eHealth interventions could be recommended in future guidelines.

\section{CONCLUSION}

Our study provides preliminary data for the future development and application of eHealth interventions for patients with MetS. The results from this systematic review and meta-analysis indicated that eHealth interventions have significant effects on body weight, BMI, WC and SBP for individuals with MetS. However, their effectiveness on TG, TC, HDL-C, LDL-C, FBG and fasting insulin is insufficient. Therefore, eHealth interventions are beneficial for improving specific anthropometric outcomes. Researchers should decide whether to use eHealth interventions according to their research objectives. Additional eHealth interventions with rigorous study designs are needed to provide robust evidence in a diverse population in the future.

Acknowledgements We thank the people who took time to become involved in the study.

Contributors ZY, DC and JS were responsible for the design of this study. DC and JS designed the search strategies and performed the literature searches. $\mathrm{DC}, \mathrm{JS}$ and $\mathrm{HZ}$ performed the review selection, and $\mathrm{DC}$ and $\mathrm{HZ}$ assessed the methodological quality of the reviews. DC, JS, LT, XW, RQ and QZ were responsible for the data extraction, analysis, and/or interpretation of the data. $Z Y$ and $D C$ drafted this manuscript in cooperation with JS, LT, XW, RQ and QZ. All authors read and approved the manuscript.

Funding This study was supported by a grant from National Health Council Scientific Research Fund of China-Key Projects of Zhejiang Provincial Major Science and Technology Program in Medicine and Health (grant number: WKJ-ZJ-1925) in 2019.

Competing interests None declared.

Patient consent for publication Not required.

Provenance and peer review Not commissioned; externally peer reviewed.

Data availability statement All data relevant to the study are included in the article or uploaded as supplementary information. No additional data available.

Open access This is an open access article distributed in accordance with the Creative Commons Attribution Non Commercial (CC BY-NC 4.0) license, which permits others to distribute, remix, adapt, build upon this work non-commercially, and license their derivative works on different terms, provided the original work is properly cited, appropriate credit is given, any changes made indicated, and the use is non-commercial. See: http://creativecommons.org/licenses/by-nc/4.0/.

\section{ORCID iDs}

Zhihong Ye http://orcid.org/0000-0001-6947-3330

Xiyi Wang http://orcid.org/0000-0002-6470-8556

\section{REFERENCES}

1 Pérez-Martínez P, Mikhailidis DP, Athyros VG, et al. Lifestyle recommendations for the prevention and management of metabolic syndrome: an international panel recommendation. Nutr Rev 2017;75:307-26.

2 Shin J-A, Lee J-H, Lim S-Y, et al. Metabolic syndrome as a predictor of type 2 diabetes, and its clinical interpretations and usefulness. $J$ Diabetes Investig 2013;4:334-43.

3 Esposito K, Chiodini P, Colao A, et al. Metabolic syndrome and risk of cancer: a systematic review and meta-analysis. Diabetes Care 2012;35:2402-11.

4 Ford ES, Li C. Metabolic syndrome and health-related quality of life among U.S. adults. Ann Epidemiol 2008;18:165-71.

5 Li R, Li W, Lun Z, et al. Prevalence of metabolic syndrome in mainland China: a meta-analysis of published studies. BMC Public Health 2016;16:296.

6 Watanabe K, Sakuraya A, Kawakami N, et al. Work-Related psychosocial factors and metabolic syndrome onset among workers: a systematic review and meta-analysis. Obes Rev 2018:19:1557-68.

7 Oliveira RGde, Guedes DP. Physical activity, sedentary behavior, cardiorespiratory fitness and metabolic syndrome in adolescents: systematic review and meta-analysis of observational evidence. PLoS One 2016;11:e0168503.

$8 \mathrm{Kim}$ J, Lee I, Lim S. Overweight or obesity in children aged 0 to 6 and the risk of adult metabolic syndrome: a systematic review and metaanalysis. J Clin Nurs 2017;26:3869-80.

9 Selvaraj P, Muthunarayanan L. Prevalence of metabolic syndrome and associated risk factors among men in a rural health centre area in Tamil Nadu. J Lifestyle Med 2019;9:44-51.

10 Huseini HF, Kianbakht S, Hajiaghaee R, et al. Anti-Hyperglycemic and anti-hypercholesterolemic effects of Aloe vera leaf gel in hyperlipidemic type 2 diabetic patients: a randomized double-blind placebo-controlled clinical trial. Planta Med 2012;78:311-6.

11 Shakib Z, Shahraki N, Razavi BM, et al. Aloe vera as an herbal medicine in the treatment of metabolic syndrome: a review. Phytother Res 2019;33:2649-60.

12 Chu P, Gotink RA, Yeh GY, et al. The effectiveness of yoga in modifying risk factors for cardiovascular disease and metabolic syndrome: a systematic review and meta-analysis of randomized controlled trials. Eur J Prev Cardiol 2016;23:291-307.

13 Esposito K, Kastorini C-M, Panagiotakos DB, et al. Mediterranean diet and metabolic syndrome: an updated systematic review. Rev Endocr Metab Disord 2013;14:255-63.

14 Eysenbach G. What is e-health? J Med Internet Res 2001;3:E20.

15 Stevenson JK, Campbell ZC, Webster AC, et al. eHealth interventions for people with chronic kidney disease. Cochrane Database Syst Rev 2019;8:CD012379. 
16 Kay M, Santos J, Takane M. mHealth: new horizons for health through mobile technologies. World Health Organization 2011;64:66-71.

17 Muellmann S, Forberger S, Möllers T, et al. Effectiveness of eHealth interventions for the promotion of physical activity in older adults: a systematic review. Prev Med 2018;108:93-110.

18 Jahangiry L, Montazeri A, Najafi M, et al. An interactive webbased intervention on nutritional status, physical activity and health-related quality of life in patient with metabolic syndrome: a randomized-controlled trial (the red ruby study). Nutr Diabetes 2017;7:e240.

19 Kim C-J, Schlenk EA, Kang S-W, et al. Effects of an Internet-based lifestyle intervention on cardio-metabolic risks and stress in Korean workers with metabolic syndrome: a controlled trial. Patient Educ Couns 2015;98:111-9.

20 Buckingham SA, Williams AJ, Morrissey K, et al. Mobile health interventions to promote physical activity and reduce sedentary behaviour in the workplace: a systematic review. Digit Health 2019;5:205520761983988.

21 Ma Y, Cheng HY, Cheng L, et al. The effectiveness of electronic health interventions on blood pressure control, self-care behavioural outcomes and psychosocial well-being in patients with hypertension: a systematic review and meta-analysis. Int J Nurs Stud 2019;92:27-46.

22 Pal K, Eastwood SV, Michie S, et al. Computer-Based diabetes selfmanagement interventions for adults with type 2 diabetes mellitus. Cochrane Database Syst Rev 2013:CD008776.

23 Moher D, Liberati A, Tetzlaff J, et al. Preferred reporting items for systematic reviews and meta-analyses: the PRISMA statement. Int $J$ Surg 2010;8:336-41.

24 Jackson N, Waters E, Guidelines for Systematic Reviews in Health Promotion and Public Health Taskforce. Criteria for the systematic review of health promotion and public health interventions. Health Promot Int 2005;20:367-74.

25 Hedges LV. Distribution theory for glass's estimator of effect size and related estimators. Journal of Educational Statistics 1981;6:107-28.

26 Cohen J. The statistical power of abnormal-social psychological research: a review. J Abnorm Soc Psychol 1962;65:145-53.

27 Higgins JPT, Thompson SG, Deeks JJ, et al. Measuring inconsistency in meta-analyses. BMJ 2003;327:557-60.

28 Lin C-H, Chiang S-L, Heitkemper MM, et al. Effects of telephonebased motivational interviewing in lifestyle modification program on reducing metabolic risks in middle-aged and older women with metabolic syndrome: a randomized controlled trial. Int J Nurs Stud 2016;60:12-23.

29 Luley C, Blaik A, Götz A, et al. Weight loss by telemonitoring of nutrition and physical activity in patients with metabolic syndrome for 1 year. J Am Coll Nutr 2014;33:363-74

30 Oh B, Cho B, Han MK, et al. The effectiveness of mobile PhoneBased care for weight control in metabolic syndrome patients: randomized controlled trial. JMIR Mhealth Uhealth 2015;3:e83.

31 Bosak KA, Yates B, Pozehl B. Effects of an Internet physical activity intervention in adults with metabolic syndrome. West $J$ Nurs Res 2010;32:5-22.

32 Fappa E, Yannakoulia M, loannidou M, et al. Telephone counseling intervention improves dietary habits and metabolic parameters of patients with the metabolic syndrome: a randomized controlled trial. Rev Diabet Stud 2012;9:36-45.

$33 \mathrm{Kim}$ J, Bea W, Lee K, et al. Effect of the telephone-delivered nutrition education on dietary intake and biochemical parameters in subjects with metabolic syndrome. Clin Nutr Res 2013;2:115-24.
34 Radhakrishnan J, Swaminathan N, Pereira N, et al. Effect of an ITsupported home-based exercise programme on metabolic syndrome in India. J Telemed Telecare 2014;20:250-8.

35 Zhang X, Wu R. Effect of Internet in young and middle-aged metabolic syndrome patients. Chinese Journal of Misdiagnosis 2011;11:8146-7.

36 Farhangi MA, Jahangiry L, Mirinazhad M-M, et al. A web-based interactive lifestyle modification program improves lipid profile and serum adiponectin concentrations in patients with metabolic syndrome: the "Red Ruby" study. Int J Diabetes Dev Ctries 2017;37:21-30.

37 Kang JS, Kang HS, Jeong Y. A web-based health promotion program for patients with metabolic syndrome. Asian Nurs Res 2014;8:82-9.

38 Park S-H, Hwang J, Choi Y-K. Effect of mobile health on obese adults: a systematic review and meta-analysis. Healthc Inform Res 2019;25:12-26.

39 Oh B, Yi G-H, Han MK, et al. Importance of active participation in obesity management through mobile health care programs: substudy of a randomized controlled trial. JMIR Mhealth Uhealth 2018;6:e2.

40 Fappa E, Yannakoulia M, Pitsavos C, et al. Lifestyle intervention in the management of metabolic syndrome: could we improve adherence issues? Nutrition 2008;24:286-91.

41 Farrell SW, Finley CE, Grundy SM. Cardiorespiratory fitness, LDL cholesterol, and CHD mortality in men. Med Sci Sports Exerc 2012;44:2132-7.

42 Jensen MD, Ryan DH, Apovian CM, et al. 2013 AHA/ACC/TOS guideline for the management of overweight and obesity in adults: a report of the American College of Cardiology/American heart association Task force on practice guidelines and the obesity Society. Circulation 2014;129:S102-38.

43 Dunkley AJ, Charles K, Gray LJ, et al. Effectiveness of interventions for reducing diabetes and cardiovascular disease risk in people with metabolic syndrome: systematic review and mixed treatment comparison meta-analysis. Diabetes Obes Metab 2012;14:616-25.

44 Wieland LSet al. Interactive computer-based interventions for weight loss or weight maintenance in overweight or obese people. Cochrane Database of Systematic Reviews 2012;8:CD007675.

45 de Koning L, Merchant AT, Pogue J, et al. Waist circumference and waist-to-hip ratio as predictors of cardiovascular events: meta-regression analysis of prospective studies. Eur Heart $J$ 2007;28:850-6.

46 Zha Pet al. Utilizing a mobile health intervention to manage hypertension in an underserved community. West $J$ Nurs Res 2019;0193945919847937.

47 Haas K, Hayoz S, Maurer-Wiesner S. Effectiveness and feasibility of a remote lifestyle intervention by dietitians for overweight and obese adults: pilot study. JMIR Mhealth Uhealth 2019;7:e12289.

48 Nolan RP, Feldman R, Dawes M, et al. Randomized controlled trial of E-Counseling for hypertension. Circulation 2018;11:e004420.

49 Sullivan AN, Lachman ME. Behavior change with fitness technology in sedentary adults: a review of the evidence for increasing physical activity. Frontiers in Public Health 2016;4:1-16.

50 Bort-Roig J, Gilson ND, Puig-Ribera A, et al. Measuring and influencing physical activity with smartphone technology: a systematic review. Sports Med 2014;44:671-86.

51 van Namen M, Prendergast L, Peiris C. Supervised lifestyle intervention for people with metabolic syndrome improves outcomes and reduces individual risk factors of metabolic syndrome: a systematic review and meta-analysis. Metabolism 2019;101:153988.

52 Quevedo Rodríguez A, Wägner AM. Mobile phone applications for diabetes management: a systematic review. Endocrinología, Diabetes y Nutrición 2019;66:330-7. 